\title{
Exotic hadron holography from anomalous dimensions
}

\section{Hilmar Forkel*}

Institut für Physik, Humboldt-Universität zu Berlin, D-12489 Berlin, Germany, and Institut für Theoretische Physik, Universität Heidelberg, D-69120 Heidelberg, Germany

E-mail: forkelephysik.hu-berlin.de

\begin{abstract}
The anomalous dimensions of hadronic interpolators contain dynamical information on the properties of the associated hadron states. We point out that they provide, in particular, a link by which gauge-invariant information on exotic contributions to hadronic wavefunctionals can be obtained from approximate gravity duals for QCD. This is demonstrated by the holographic description of a dominant tetraquark component in the lightest scalar mesons.
\end{abstract}

Xth Quark Confinement and the Hadron Spectrum,

October 8-12, 2012

TUM Campus Garching, Munich, Germany

${ }^{*}$ Speaker. 
While the anomalous dimensions of color-singlet operators play a central role in the original, conformal versions of the gauge/string correspondence [1], they have only recently begun to enter holographic approaches to QCD. In particular, anomalous dimensions of hadronic interpolators were implemented to provide an AdS/QCD [2] description of quark correlations inside hadrons $[3,4]$ which can have a significant and in exotic cases even striking impact on the hadron properties. Although multiquark components in hadronic wave functionals are typically gauge dependent, a holographic description is still possible because the five-dimensional bulk modes are dual to the interpolators of the corresponding hadrons. Hence the anomalous dimensions of these interpolators import gauge-invariant information on their quark content and couplings, and thus on the multiquark correlations in the corresponding hadron, as bulk-mode mass corrections into the gravity dual.

This AdS/QCD representation of multiquark effects was originally introduced to describe diquark correlations in baryons [3]. It changes the resulting light-quark baryon excitation spectrum into

$$
M_{n, L}^{2}=4 \lambda^{2}\left(n+L+\frac{3}{2}\right)-2\left(M_{\Delta}^{2}-M_{N}^{2}\right) \kappa
$$

(where $\lambda$ is the IR scale of the "metric soft-wall" gravity dual [5] while $n(L)$ denotes the radial (angular momentum) excitation level). The second term, proportional to the baryon's "good-diquark fraction" $\kappa$, is generated by suitable anomalous dimensions for the QCD nucleon interpolators. Equation (1) describes the linear square-mass trajectories of the over 40 measured nucleon and delta (with $\kappa=0$ ) resonances with unprecendented accuracy. The dual mode solutions further reveal that baryons with larger $\kappa$ have a smaller size.

Encouraged by these results, the anomalous-dimension-induced representation of multiquark correlations was then applied to the more challenging holographic description of exotic hadrons with a non-standard (valence) quark content. The light scalar meson sector [6] with its expected tetraquark component [7] was examined in Ref. [4]. The radial bulk equation for the modes dual to the scalars can be written as the Sturm-Liouville problem $\left[-\partial_{z}^{2}+V(z)\right] \phi(q, z)=q^{2} \phi(q, z)$. In the dilaton soft-wall gravity dual [8] without anomalous-dimension contributions, the potential $V$ has the form

$$
V(z)=\left(\frac{15}{4}+m_{5}^{2} R^{2}\right) \frac{1}{z^{2}}+\lambda^{2}\left(\lambda^{2} z^{2}+2\right) .
$$

The anomalous dimension $\gamma(z)$ of the tetraquark interpolator $J_{\bar{q}^{2} q^{2}}$ (i.e. the local four-quark operator which most strongly couples to the tetraquark state) with scaling dimension $\Delta_{\bar{q}^{2} q^{2}}=6+\gamma(z)$ adds the universal contribution

$$
\Delta V(z)=\gamma(z)[\gamma(z)+8] \frac{1}{z^{2}}
$$

to the potential (2) with $m_{5}^{2} R^{2}=12$. Eq. (3) implies the crucial lower bound $\Delta V(z) \geq-16 / z^{2}$ which holds for any $\gamma$ and prevents the collapse of the dual modes into the $\mathrm{AdS}_{5}$ boundary. This bound is saturated by $\gamma \equiv-4$ and therefore determines the lightest tetraquark mass

$$
M_{\bar{q}^{2} q^{2}, 0} \geq M_{\Delta=2,0}=2 \lambda
$$

which the anomalous-dimension-induced holographic binding mechanism can produce. Moreover, for constant values $-4<\gamma<-3$ the tetraquark ground state is lighter than its $\bar{q} q$ counterpart. Since 
$\gamma$ only enters through the mass term of the bulk mode which is model-independently prescribed by the AdS/CFT dictionary, the correction (3) and the associated binding mechanism will arise in other AdS/QCD duals as well.

To estimate the quantitative impact of the anomalous-dimension contribution $\Delta V$ (until direct QCD information on the RG flow of $\gamma$ will eventually become available and fix $\Delta V$ uniquely), a typical power ansatz $\gamma(z)=-a z^{\eta}+b z^{\kappa}$ can be adopted. Its coefficients turn out to be tightly constrained by consistency and stability requirements but can still produce almost maximal groundstate binding [4]. The latter drives the mass $M_{\bar{q}^{2} q^{2}, 0}$ of the lightest tetraquark from $\sim 40 \%$ above (for $\gamma \equiv 0$ ) down to $\sim 20 \%$ below the $\bar{q} q$ ground-state mass $M_{q \bar{q}, 0}=\sqrt{6} \lambda$. The resulting masses $M_{\bar{q}^{2} q^{2}, n}$ of the tetraquark excitations get pushed beyond the corresponding $M_{\bar{q} q, n}$ from around $n \gtrsim 2$. The higher-lying radial tetraquark excitations will therefore likely be broad enough to prevent the appearance of supernumeral states in the scalar meson spectrum.

It should be interesting to extend the anomalous-dimension-based holographic description of non-valence quark components to other exotics, including heavy tetraquarks, pentaquarks and hybrids. Moreover, anomalous-dimension-induced corrections also encode other aspects of hadronic structure which largely remain to be explored.

\section{Acknowledgments}

It is a pleasure to thank the organizers for a very informative and enjoyable conference.

\section{References}

[1] O. Aharony et al., Large-N field theories, string theory and gravity, Phys. Rep. 323 (2000) 183.

[2] Y. Kim and D. Yi, Holography at work for nuclear and hadron physics, Adv. High Energy Phys. 2011 (2011) 259025; S.J. Brodsky and G.F. de Téramond, AdS/CFT and Light-Front QCD, arXiv:0802.0514.

[3] H. Forkel and E. Klempt, Diquark correlations in baryon spectroscopy and holographic QCD, Phys. Lett. B 679 (2009) 77.

[4] H. Forkel, Light scalar tetraquarks from a holographic perspective, Phys. Lett. B 694 (2010) 252; Multiquark correlations in light mesons and baryons from holographic QCD, AIP Conf. Proc. 1388 (2011) 182 [arXiv:1103.3902].

[5] H. Forkel, M. Beyer and T. Frederico, Linear square-mass trajectories of radially and orbitally excited hadrons in holographic QCD, JHEP 07 (2007) 077; Linear meson and baryon trajectories in AdS/QCD, Intl. J.Mod. Phys. E 16 (2007) 2794.

[6] E. Klempt and A. Zaitsev, Glueballs, hybrids, multiquarks - Experimental facts versus QCD inspired concepts, Phys. Rep. 454, 1 (2007); C. Amsler and N.A. Törnqvist, Mesons beyond the naive quark model, Phys. Rep. 389, 61 (2004); D.V. Bugg, Four sorts of meson, Phys. Rept. 397, 257 (2004); E. Ruiz Arriola and W. Broniowski, Scalar-isoscalar states in the large- $N_{c}$ Regge approach, Phys. Rev. D. 81 (2010) 054009.

[7] R.L. Jaffe, Phenomenology of $Q^{2} \bar{Q}^{2}$ mesons, Phys. Rev. D 15, 267, 281 (1977); J.R. Peláez, Nature of light scalar mesons from their large- $N_{c}$ behavior, Phys. Rev. Lett. 92 (2004) 102001.

[8] A. Karch, E. Katz, D.T. Son and M.A. Stephanov, Linear Confinement and AdS/QCD, Phys. Rev. D 74 (2006) 015005. 